\title{
Ocorrência de Automedicação na população Brasileira como estratégia preventiva ao
}

\section{SARS-CoV-2}

Occurrence of Self-medication in the Brazilian population as a preventive strategy for SARS-CoV-2

Aparición de la automedicación en la población brasileña como estrategia preventiva del SARS-

CoV-2

Maria Nathalya Costa Souza

ORCID: https://orcid.org/0000-0003-0545-5795 Faculdade de Medicina Estácio de Juazeiro do Norte, Brasil E-mail: nathalya535@hotmail.com

Isadora Ellen Feitoza Ricardino ORCID: https://orcid.org/0000-0002-9497-6957 Faculdade de Medicina Estácio de Juazeiro do Norte, Brasil E-mail: isadoraricardino@gmail.com

Kennedy Sampaio

ORCID: https://orcid.org/0000-0001-6894-4217 Centro Universitário Dr. Leão Sampaio, Brasil E-mail: kennedysampaio062@gmail.com

Marcolino Ribeiro Silva

ORCID: https://orcid.org/0000-0003-3792-260X Centro Universitário Dr. Leão Sampaio, Brasil

E-mail: ribeirom996@gmail.com

Ana Patrícia Gomes de Lima

ORCID: https://orcid.org/0000-0002-0853-0490

Centro Universitário de Juazeiro do Norte Brasil E-mail: pgl.patricia1994@gmail.com

Danilo Leite Fernandes

ORCID: https://orcid.org/0000-0003-2671-3351 Instituto Federal de Educação, Ciência e Tecnologia do Ceará, Brasil E-mail: danilofernandes@ hotmail.com

Adalberto Cruz Sampaio

ORCID: https://orcid.org/0000-0003-4062-2472 Faculdade de Medicina Estácio de Juazeiro do Norte, Brasil E-mail: adalbertocruzjua@gmail.com

Andréa Couto Feitosa

ORCID: https://orcid.org/0000-0001-8600-9126 Centro Universitário Dr. Leão Sampaio, Brasil

E-mail: andreafeitosa@leaosampaio.edu.br

Alessandra Bezerra de Brito

ORCID: https://orcid.org/0000-0003-4511-379X Centro Universitário Dr. Leão Sampaio, Brasil E-mail: abbrito2020@gmail.com

Tarciana Oliveira Guedes

ORCID: https://orcid.org/0000-0002-8200-1298

Centro Universitário Saúde do ABC, Brasil E-mail: tarciana-guedes@hotmail.com

Magaly Lima Mota

ORCID: https://orcid.org/0000-0002-6712-6236

Centro Universitário Dr. Leão Sampaio, Brasil E-mail: magaly_lm@yahoo.com.br

\section{Resumo}

Objetivou-se identificar a existência da automedicação por populares com a finalidade de prevenção ao SARS-CoV-2 e analisar os potenciais agravamentos deste uso ao organismo humano. Trata-se de uma pesquisa descritiva e analítica, de natureza quantitativa, onde a coleta de dados desse estudo foi realizada através de um formulário semiestruturado, disponibilizado de forma eletrônica, utilizando a ferramenta Google Forms. Os critérios de inclusão compreendem: usuário de rede social e que responderam o formulário no período estabelecido pelos pesquisadores. Há a prevalência de participantes do sexo feminino e portadores de ensino fundamental e superior completo, no qual não houve predomínio da prática de automedicação. Os maiores índices de automedicação aos fármacos Ivermectina e 
Azitromicina, a maioria dos indivíduos adquiriram esses medicamentos através da farmácia comercial. Portanto é importante ressaltar que não se deve ingerir fármacos sem a orientação médica, o que demostra a importância de que os profissionais de saúde bem como os veículos midiáticos, conscientizem a população sobre os cuidados que devem ser tomados e os riscos que eles podem sofrer ao se automedicar.

Palavras-chave: COVID-19; Automedicação; Fármacos; Tratamento.

\begin{abstract}
The objective was to identify the existence of self-medication by people with the purpose of preventing SARS-CoV-2 and to analyze the potential worsening of this use to the human organism. It is a descriptive and analytical research, of a quantitative nature, where the data collection of this study was carried out through a semi-structured form, made available electronically, using the Google Forms tool. Inclusion criteria include: social network user and who answered the form in the period established by the researchers. There is a prevalence of female participants and people with primary and higher education, in which there was no predominance of self-medication. The highest rates of self-medication to the drugs Ivermectin and Azithromycin, most individuals purchased these drugs through the commercial pharmacy. Therefore, it is important to emphasize that drugs should not be ingested without medical guidance, which demonstrates the importance of health professionals as well as media vehicles, making the population aware of the care that should be taken and the risks that they may suffer when self-medicating.
\end{abstract}

Keywords: COVID-19; Self-medication; Drugs; Treatment.

\title{
Resumen
}

El objetivo fue identificar la existencia de automedicación por parte de las personas con el propósito de prevenir el SARS-CoV-2 y analizar el potencial agravamiento de este uso en el organismo humano. Se trata de una investigación descriptiva y analítica, de carácter cuantitativo, donde la recogida de datos de este estudio se realizó a través de un formulario semiestructurado, puesto a disposición de forma electrónica, utilizando la herramienta Google Forms. Los criterios de inclusión incluyen: usuario de la red social y que contestó el formulario en el plazo establecido por los investigadores. Existe una prevalencia de mujeres participantes y personas con educación primaria y superior, en las que no hubo predominio de la automedicación. Las tasas más altas de automedicación con los medicamentos ivermectina y azitromicina, la mayoría de las personas compraron estos medicamentos a través de la farmacia comercial. Por ello, es importante resaltar que los medicamentos no deben ser ingeridos sin orientación médica, lo que demuestra la importancia de los profesionales de la salud así como de vehículos mediáticos, concienciando a la población de los cuidados que se deben tomar y los riesgos que pueden sufrir cuando se auto -medicación.

Palabras clave: COVID-19; Automedicación; Drogas; Tratamiento.

\section{Introdução}

O novo SARS-CoV-2, agente causal da COVID-19, é um beta coronavírus ( $\beta C$ VV) assim como o MERS-CoV e o SARS-CoV com patogênese semelhante à induzida pelos vírus anteriormente citados, causando mais comumente pneumonia aguda, febre, tosse seca e dispneia. A detecção rápida da COVID-19 é essencial para o controle dos surtos em comunidades e unidades hospitalares. Para isso, um dos testes de diagnóstico considerado padrão é a Reação em Cadeia da Polimerase em Transcrição Reversa (RT-PCR), sendo a investigação do RNA polimerase dependente de RNA (RdRp) o protocolo recomendado para confirmar o resultado positivo (Li, Bai \& Hashikawa, 2020).

Ainda não existem até o presente momento vacinas ou fármacos antivirais específicos para prevenir ou tratar a COVID-19. Dessa forma, as pessoas infectadas devem receber cuidados de saúde para aliviar os sintomas, e, em casos onde apresentam a Síndrome Respiratória Aguda Grave (SARS), elas devem ser hospitalizadas para maiores cuidados de suporte médico. Atualmente, estão sendo investigadas possíveis vacinas e alguns tratamentos medicamentosos específicos, com testes randomizados in vivo para avaliar seus potenciais efeitos contra o SARS-CoV-2 (Zhai et al., 2020).

Assim, a prevenção consiste em proteger a si e aos outros, higienizando frequentemente as mãos, cobrir a tosse com a parte interior do cotovelo ou lenço e manter uma distância de pelo menos 1 metro das pessoas que estão tossindo ou espirrando. Entretanto, mediante as incertezas que emergiram com a pandemia pelo novo coronavírus, tem-se observado uma intensificação comportamental da população em se automedicar, acreditando que assim estarão mais seguras (WHO, 2020).

$\mathrm{O}$ aumento da procura e consumação de suplementos alimentares, como vitaminas e minerais, para fortalecer a imunidade e prevenir a infecção pelo vírus, assim como, fármacos com propriedade antiparasitária ou antibiótica tem ocorrido 
consideravelmente durantes esta pandemia (Pereira, 2020). Dessa forma, o presente estudo tem como objetivo identificar a existência da automedicação por populares com a finalidade de prevenção ao SARS-CoV-2 e analisar os potenciais agravamentos deste uso ao organismo humano.

\section{Metodologia}

Trata-se de uma pesquisa descritiva e analítica, de natureza quantitativa, onde este método caracteriza-se pelo emprego da quantificação, tanto nas modalidades de coleta de informações, quanto na filtragem dessas através de técnicas estatísticas, desde as mais simples até as mais complexas (Pereira. et al., 2018). A coleta de dados foi realizada durante o mês de julho de 2020. Considerando o momento atual de pandemia pela COVID-19, com as medidas sanitárias colocadas à população, tais como de isolamento social que prevê diminuição do contato físico pessoal, a coleta de dados desse estudo atendeu às orientações das autoridades políticas e da saúde, sendo realizada de modo virtual, visando preservar a saúde dos envolvidos e reduzir os riscos de contaminação, respeitando as diretrizes previstas pela Organização Mundial de Saúde.

Assim, os participantes não foram expostos ao contato físico, havendo a coleta de dados por meio da plataforma virtual WhatsApp, através de um formulário semiestruturado, disponibilizado de forma eletrônica ao usuário da rede social, utilizando a ferramenta Google Forms. A amostra foi obtida por meio dos seguintes critérios de inclusão: usuário de rede social e que responderam o formulário no período estabelecido pelos pesquisadores, totalizando 509 entrevistados residentes nos Estados: CE, PE, PI, SP, MT, MA, PB, ES, BA, RN, RO e RJ. Como critérios de exclusão: pessoas que não utilizam rede social e que não responderam o formulário no período estabelecido. Os dados obtidos foram analisados em percentuais e apresentados em gráficos, utilizando a ferramenta Excel da Microsoft.

A pesquisa atendeu aos preceitos éticos estabelecidos pela Resolução No 510, de Abril de 2016, do Conselho Nacional de Saúde que dispõe sobre as normas aplicáveis a pesquisas em ciência humanas e sociais (Brasil, 2016). A mesma foi aprovada pelo Comitê de Ética em Pesquisa do Centro Universitário Doutor Leão Sampaio com protocolo CAAE $\mathrm{n}^{\circ}$. 38096720.1 .0000 .5048 .

\section{Resultados}

Após a coleta de dados foi possível constatar que, dos 509 entrevistados, a maioria pertence ao sexo feminino (74,5\%) comparado ao sexo masculino. E, quanto ao grau de escolaridade, o predomínio foi de ensino fundamental completo (44,4\%), seguido por ensino superior $(22,4 \%)$, conforme apresentado na Figura 1. 
Figura 1. Caracterização dos entrevistados quanto ao sexo e o grau de escolaridade, Brasil, 2020.

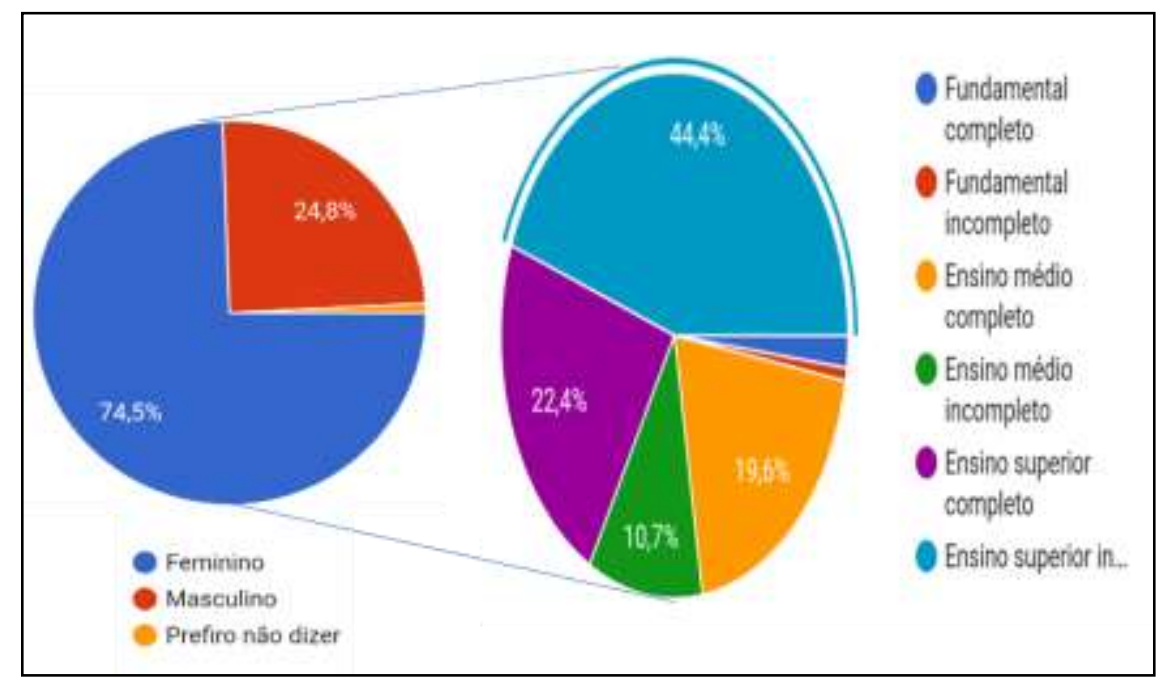

Fonte: Autores (2020).

Quando indagados sobre a realização de automedicação no intuito de prevenir ou tratar a infecção pelo SARS-CoV-2, a maioria dos participantes $(69,2 \%)$ relatou não ter se automedicado. Dos que realizaram automedicação, o fármaco de maior uso foi Ivermectina (52,8\%), seguido por Azitromicina (14,2\%), como expresso na Figura 2.

Figura 2. Prevalência de automedicação e fármacos utilizados pelos entrevistados durante a pandemia da COVID-19, Brasil, 2020.

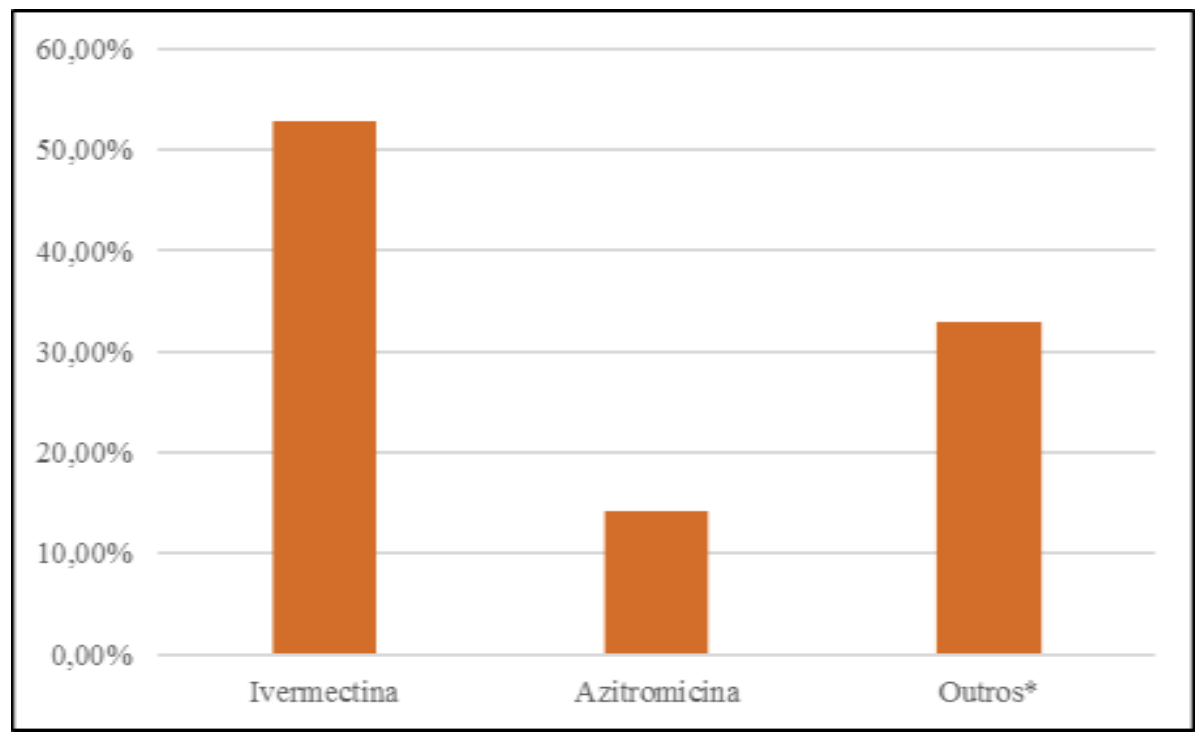

*Hidroxicloroquina, Nitazoxanida, Loratadina. Fonte: Autores (2020).

Em relação à ingestão de suplementos vitamínicos com intuito de fortalecer a imunidade durante a pandemia da COVID-19, constatou-se que as Vitaminas C e D foram as mais utilizadas pelos participantes, com 66,4\% e 10,9\%, respectivamente (Figura 3). 
Figura 3. Suplementos vitamínicos de maior utilização pelos entrevistados durante a pandemia da COVID-19, Brasil, 2020.

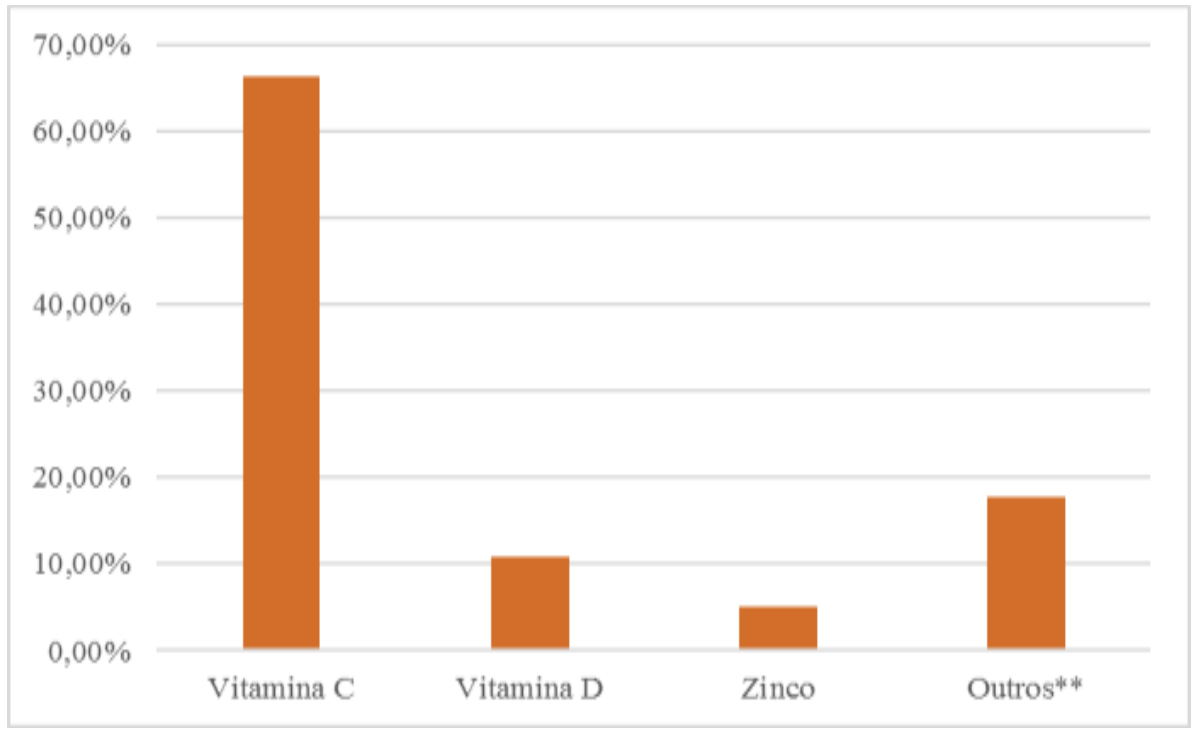

**Ferro, Polivitamínico. Fonte: Autores (2020).

A respeito da origem dos fármacos, a grande maioria dos entrevistados $(91,2 \%)$ afirmou que adquiriram em farmácia comercial, e, quando indagados sobre o conhecimento dos potenciais riscos da automedicação bem como dos efeitos colaterais que podem surgir, houve uma predominância de pessoas que afirmaram conhecer os efeitos adversos, conforme apresentado na Figura 4.

Figura 4. Origem de aquisição dos fármacos e autoconhecimento dos entrevistados sobre os efeitos adversos durante a pandemia da COVID-19, Brasil, 2020.

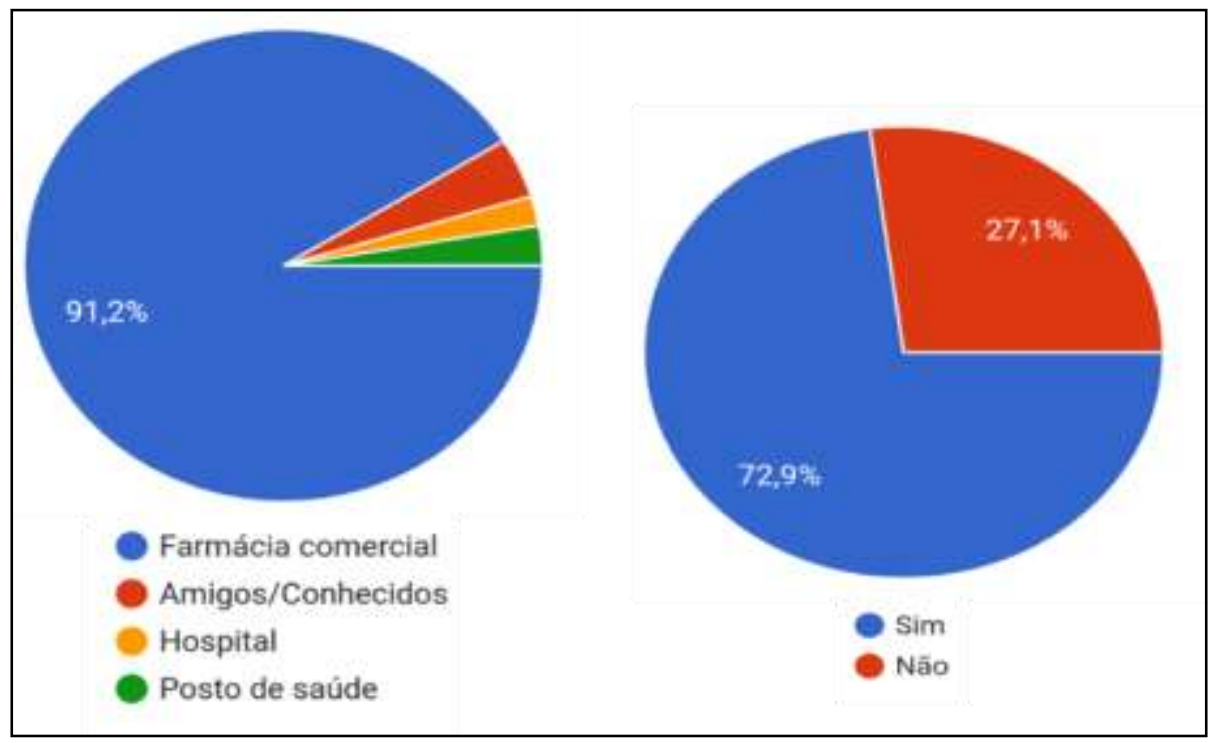

Fonte: Autores (2020).

De acordo com os dados coletados 72,9\% das pessoas que responderam ao formulário conhecem os efeitos nocivos que esses medicamentos possuem enquanto $27,1 \%$ desconhecem sobre os mesmos, sendo de grande relevância a compreensão e prevenção de efeitos adversos ou outros problemas relacionados a medicamentos. 


\section{Discussão}

Verifica-se nesse estudo uma prevalência de participantes do sexo feminino e portadores de ensino fundamental e superior completo, no qual não houve predomínio da prática de automedicação. Provavelmente, esse fato pode estar relacionado ao fato de que, geralmente, as mulheres quando comparada aos homens, são mais atentas quanto ao seu bem-estar e a manutenção da sua saúde, levando-as a procurar mais informações e serviços na atenção primária, sendo os homens mais resistentes neste aspecto.

Uma pesquisa sobre as características principais dos usuários dos serviços de atenção primária à saúde no Brasil foi entrevistada 8.676 usuários, sendo a grande maioria (75,8\%) constituída por mulheres (Guibu et al., 2017). Analisando as características dos usuários em um serviço de uma Unidade Básica de Saúde (UBS) percebeu-se que o percentual de clientes do sexo feminino (54,65\%) superou o percentual masculino, sendo o público adulto (43\%) (Friedlander, Guimarães \& Fabichacki, 2016).

Quanto aos participantes que realizaram a automedicação no presente período pandêmico, houve destaque para os fármacos Ivermectina e Azitromicina, e, em relação ao suplemento vitamínico, houve prevalência das vitaminas $\mathrm{C}$ e $\mathrm{D}$, sendo estes os mais utilizados para prevenir ou tratar infecções pelo SARS-CoV-2.

A atual pandemia da doença de coronavírus 2019 (COVID-19) trouxe inúmeros desafios para os sistemas de saúde em quase todos os países do mundo. Atualmente, não existem vacinas eficazes comprovadas ou agentes terapêuticos contra o vírus, e, as pesquisas em desenvolvimento sugerem uma considerável lista de medicamentos com efeitos farmacológicos apropriados e eficácia terapêutica no tratamento de pacientes com a COVID-19. Esses fármacos e agentes terapêuticos incluem: agentes antivirais (remdesivir, hidroxicloroquina, cloroquina, lopinavir, umifenovir, favipiravir e oseltamivir) e agentes de suporte (ácido ascórbico, azitromicina, corticosteróides, óxido nítrico, antagonistas da IL-6), entre outros (Wu et al., 2020).

A Ivermectina é um agente antiparasitário de amplo espectro de ação que apresenta, assim como outros grupos de fármacos, atividade antiviral contra uma vasta gama de vírus in vitro, que se acredita ser devido à dependência de muitos vírus de RNA no IMP $\alpha$ / $\beta 1$. Aponta-se que as proteínas do SARS-CoV-2 possuem um papel para IMP $\alpha$ / $\beta 1$ durante o fechamento do nucleocitoplasmático durante a infecção. Essa evidência sugere a atividade inibidora do transporte nuclear da Iverrmectina pode ser eficaz contra o novo coronavírus. Em testes realizados in vitro foi possível observar redução de aproximadamente 5000 vezes o RNA viral em amostras tratadas com Ivermectina contra o SARS-CoV-2 em 48 horas, vale salientar que não foi registrado toxicidade nos testes (Caly et al., 2020).

Outro estudo relatou que a Ivermectina não é capaz de atingir a $\mathrm{IC}_{50}$ mesmo utilizando doses 10 vezes maiores que a dose aprovada ( $200 \mu \mathrm{g} / \mathrm{kg}$ ) ou após doses repetidas, podendo chegar a ser tóxica ao organismo humano. Esse fato demostra que a readaptação de medicamentos para o tratamento da COVID-19 é uma prática válida, mas só pode ser viável se a segurança do uso desse fármaco for estabelecida em níveis de doses que sejam eficazes, reafirmando a importância de que estudos in vitro sejam realizados em concentrações que sejam relevantes clinicamente (Schmith, Zhou \& Lohmer, 2020).

A Azitromicina (AZT) é um antibiótico da classe dos macrolídeos usado no tratamento de bronquite, pneumonia, infecções sexualmente transmissíveis, cutâneas e infecção por Mycobacterium avium (Choudhary \& Sharma, 2020). Além disso, quando testado in vitro apresentou atividade antiviral contra os vírus Zika e Ebola e para prevenir infecções graves do trato respiratório quando tratadas em pacientes que sofrem de infecção viral (Bacharier et al., 2015; Retallack et al., 2016).

O mecanismo de ação da AZT envolve a ligação do princípio ativo do fármaco ao rRNA na porção 23S da subunidade ribossômica 50S dos microrganismos, o que inibe a síntese proteica bacteriana e impede a montagem da subunidade 50S. Investigações demostram a atividade antiviral in vitro da AZT contra patógenos virais (exceto H1N1), com $\mathrm{IC}_{50}$ variando aproximadamente 1-6 $\mu \mathrm{M}$ (Damle et al., 2020). 
Foi observado que associação entre Hidroxicloroquina e Azitromicina possuía efeitos sinérgicos contra o SARS-CoV2 in vitro, já que a AZT também é uma base fraca que se acumula nos endossomos com um efeito alcalinizante equivalente à Hidroxicloroquina, além de possuir também atividades imunomoduladoras. Vale ressaltar que os efeitos adversos observados no uso da Hidroxicloroquina devem ser bem avaliados quando usados em associação com a AZT, pois esses riscos podem ser ainda mais potencializados com a utilização concomitante desses dois fármacos (Felsenstein et al., 2020).

Devido à capacidade e potencial para estimular à resposta imunológica e à sua disponibilidade como medicamentos de venda livre, o ácido ascórbico (vitamina C), o zinco, a vitamina D e N-acetilcisteína foram sugeridos como elementos úteis para prevenção ou tratamento da COVID-19 (Bauer et al., 2020). A vitamina C constitui um nutriente essencial com papéis significativos no corpo humano, como a neutralização dos radicais livres, ajuda a prevenir ou reverter os danos celulares como um potente agente antioxidante e está relacionada em alguns processos biológicos, muitos dos quais estão associados à saúde imunológica (Carr \& Maggini, 2017).

Além disso, a vitamina $\mathrm{C}$ parece ser eficaz como agente antiviral, especialmente contra o vírus influenza. Muitos estudos mostraram que a vitamina $\mathrm{C}$ afeta positivamente o desenvolvimento e maturação de linfócitos T e células NK (Natural Killer) envolvidos na resposta imune a agentes virais. Também contribui à inibição de espécies reativas de oxigênio (ERO), produção e remodelação da rede de citocinas típicas da síndrome inflamatória sistêmica (Van et al., 2018).

Estudos in vitro e in vivo em aves mostraram que a vitamina $\mathrm{C}$ apresentou proteção contra a infecção por coronavírus aviário. Em humanos constatou-se que a vitamina $\mathrm{C}$ pode diminuir a suscetibilidade a pneumonia e infecções respiratórias virais (Zhang \& Liu, 2020). Avaliação de vitamina C para o tratamento de pacientes hospitalizados e pacientes críticos mostraram resultados variados sobre mortalidade, tempo de permanência na unidade de terapia intensiva e duração da ventilação mecânica (Carr, 2019). No entanto, o impacto da vitamina C no tratamento de pacientes com COVID-19 ainda não está totalmente esclarecido e novos ensaios clínicos estão em andamento na China e nos Estados Unidos (Bauer et al., 2020).

A vitamina D é encontrada em alimentos como laticínios, cereais e peixes oleosos, sendo convertida em sua forma biologicamente ativa 25-hidroxivitamina D na pele através do efeito da radiação ultravioleta em 7-desidrocolesterol. Testes in vitro mostraram que a vitamina $\mathrm{D}$ tem efeitos imunomoduladores, como inibição de células apresentadoras de antígenos, efeitos antiproliferativos de Células $\mathrm{T}$, modulando a expressão e secreção de interferon do tipo 1 e inibição da expressão de citocinas pró-inflamatórias (IL-6 e TNF alfa) (Grant et al., 2020).

A deficiência de vitamina $\mathrm{D}$ tem sido associada a uma maior incidência de infecções respiratórias agudas, com uma ligação hipotética entre a sazonalidade da gripe e deficiência de vitamina D. Foi constatado que a deficiência da vitamina D em bezerros aumentou a suscetibilidade à infecção por coronavírus bovino, que podem ter implicações na infecção por COVID-19 em humanos (Zhang \& Liu, 2020).

Um estudo recente recomendou que os pacientes em risco para COVID-19 consideram iniciar a suplementação diária de vitamina D para aumentar as concentrações séricas de 25-hidroxivitamina D com objetivo de reduzir o risco de infecção (Grant et al., 2020). Embora essa estratégia seja improvável que cause danos, não foi especificamente avaliado para prevenção da infecção por COVID-19 e mais estudos são necessários antes de ser recomendado aos pacientes (Bauer et al., 2020).

A administração de medicamentos candidatos com efeito antiviral logo após o início dos sintomas podem reduzir a infecciosidade a outros indivíduos, diminuindo a disseminação viral nas secreções respiratórias de pacientes infectados com SARS-CoV-2 que geralmente atingem o pico entre 5 e 6 dias após o início dos sintomas e dura até 14 dias (Mitja \& Clotet, 2020).

Além disso, devido ao seu amplo perfil de segurança, menos efeitos colaterais farmacológicos e fácil disponibilidade, fitoquímicos, como flavonóides, emodina e resveratrol com eficácia terapêutica postulada contra a infecção por SARS-CoV-2 podem ser usadas por grandes populações para profilaxia pré e pós-exposição da infecção por SARS-CoV-2 e em doença de 
COVID-19. Finalmente, no caso urgente da aceleração da pandemia da COVID-19, implementação de profilaxia e tratamento farmacológico antiviral de grandes populações tem vários requisitos de alta eficácia, segurança de medicamentos, alta disponibilidade e economia (Dwight et al., 2020).

Embora os fármacos sejam produzidos, indiscutivelmente sob critérios de proteção e segurança, é comum o risco associado ao seu uso a partir de motivos diversos que expõem as pessoas a efeitos indesejados. A utilização de medicamentos em situações não indicadas ou em circunstâncias que desrespeitem os critérios de uso racional pode provocar danos como a ocorrência de intoxicações. Logo é vista a importância da compressão e conhecimento frente aos efeitos adversos que podem vir a ocorrer, promovida a partir do uso racional de medicamentos (Lopes, 2018).

A farmácia comercial se configura como um dos primeiros estabelecimentos de saúde ao qual o paciente tem contato, tornando o profissinal farmacêutico fundamental para a promoção do uso racional de medicamentos. Devido ao alto custo das consultas médicas aliada à demora do Sistema Único de Saúde em dispoibilizar consultas, a prescrição de medicamentos por profissionais habilitados dá espaço para a prática da automedicação entre a população, facilitada pelo acesso rápido ao medicamento nas farmácias comerciais (Arrabal \& Salvi, 2018).

\section{Conclusão}

O uso de suplementos vitamínicos auxilia no aumento da imunidade corporal e na prevenção do contágio ao SARSCoV-2, porém, não há comprovações quanto ao uso no tratamento da respectiva virose. O uso dos fármacos supracitados não é indicado, visto que não foi constatada a eficácia dos mesmos e, em alguns casos, podem causar riscos à saúde humana. A maioria das pessoas entrevistadas é do sexo feminino e com escolaridade avançada relataram que não realizaram a prática de automedicação o que é recomendado.

É compreensível o temor em relação a saúde, mas é importante ressaltar que não se deve ingerir fármacos sem prescrição de um especialista, e ainda sem se ter conhecimento sobre os efeitos colaterais que eles podem apresentar. É de suma importância que as indústrias farmacêuticas realizem pesquisas mais abrangentes, de âmbito nacional e internacional, para entender o perfil de medicamentos mais utilizados nessa fase de confinamento e, por meio dos veículos midiáticos, disponibilizar informações importantes sobre esses fármacos, ao mesmo tempo em que conscientizam a população em geral sobre os cuidados que devem ser tomados e os riscos que o cidadão sofre ao se automedicar.

Logo, posteriores trabalhos, perante esse tema deverão levar em consideração os mais diversos problemas que a automedicação pode vir a trazer, bem como os riscos da ingestão indiscriminada de antibióticos e das possíveis interações medicamentosas que podem vir a ocorrer, é importante salientar o cuidado com o processo de medicação e a filtragem de informações divulgadas pela mídia frente aos cuidados que devem ser realizados diante o SARS-CoV2.

\section{Referências}

De Lima Fontes, F. L., de Lavor Delgado, M. L., de Assis, R. J. S., Lima, I. C., \& Lima, L. S. (2020). Alinhamento geopolítico entre os governos americano e brasileiro quanto ao uso da cloroquina/hidroxicloroquina: repercussões no enfrentamento da covid-19. International Journal of Health Management Review, $6(2), 1-17$.

Júnior, J. M. A., \& de Oliveira Salvi, J. (2018). Fatores associados à automedicação em uma farmácia comunitária de Ouro Preto do Oeste, Rondônia. Acta Biomedica Brasiliensia, 9(2), 107-16.

Bacharier, L. B., Guilbert, T. W., Mauger, D. T., Boehmer, S., Beigelman, A., Fitzpatrick, A. M., \& Martinez, F. D. (2015). Early administration of azithromycin and prevention of severe lower respiratory tract illnesses in preschool children with a history of such illnesse s: a randomized clinical trial. Jama, 314(19), 2034-44.

Bauer, S. R., Kapoor, A., Rath, M., \& Thomas, S. A. (2020). What is the role of supplementation with ascorbic acid, zinc, vitamin D, or N-acetylcysteine for prevention or treatment of COVID-19? Cleveland Clinic journal of medicine. 
Resolução $n^{\circ}$ 510, de 07 de abril de 2016. (2016). Dispõe sobre as normas aplicáveis a pesquisas em Ciências Humanas e Sociais cujos procedimentos metodológicos envolvam a utilização de dados diretamente obtidos com os participantes ou de informações identificáveis ou que possam acarretar riscos maiores do que os existentes na vida cotidiana, na forma definida nesta Resolução. Brasília. http://conselho.saude.gov.br/resolucoes/2016/Reso510.pdf.

Caly, L., Druce, J. D., Catton, M. G., Jans, D. A., \& Wagstaff, K. M. (2020). The FDA-approved drug ivermectin inhibits the replication of SARS-CoV-2 in vitro. Antiviral research, 104787.

Carr, A. C. (2019). Vitamin C administration in the critically ill: a summary of recent meta-analyses. Critical Care, $23(1), 265$.

Carr, A. C., \& Maggini, S. (2017). Vitamin C and immune function. Nutrients, 9(11), 1211.

Choudhary, R., \& Sharma, A. K. (2020). Potential use of hydroxychloroquine, ivermectin and azithromycin drugs in fighting COVID-19: trends, scope and relevance. New Microbes and New Infections, 100684.

Damle, B., Vourvahis, M., Wang, E., Leaney, J., \& Corrigan, B. (2020). Clinical Pharmacology Perspectives on the Antiviral Activity of Azithromycin and Use in COVID-19. Clinical Pharmacology \& Therapeutics. 108(2), 201-11.

McKee, D. L., Sternberg, A., Stange, U., Laufer, S., \& Naujokat, C. (2020). Candidate drugs against SARS-CoV-2 and COVID-19. Pharmacological Research. 157. 104859.

Felsenstein, S., Herbert, J. A., McNamara, P. S., \& Hedrich, C. M. (2020). COVID-19: immunology and treatment options. Clinical Immunology, 215. 108448 .

Friedlander, M. R., Guimarães, C. R. R., \& Fabichacki, E. (2016). O perfil do usuário de uma Unidade Básica de Saúde integrada a uma Faculdade Privada. Revista Desafios, 3(2), 3-13.

Grant, W. B., Lahore, H., McDonnell, S. L., Baggerly, C. A., French, C. B., Aliano, J. L., \& Bhattoa, H. P. (2020). Evidence that vitamin D supplementation could reduce risk of influenza and COVID-19 infections and deaths. Nutrients, 12(4), 988.

Guibu, I. A., Moraes, J. C. D., Guerra Junior, A. A., Costa, E. A., Acurcio, F. D. A., Costa, K. S., \& Álvares, J. (2017). Características principais dos usuários dos serviços de atenção primária à saúde no Brasil. Revista de Saúde Pública, 51, 17s.

Li, Y. C., Bai, W. Z., \& Hashikawa, T. (2020). The neuroinvasive potential of SARS-CoV2 may play a role in the respiratory failure of COVID-19 patients. Journal of medical virology, 92(6), 552-55.

Lopes, M. M. S. N. (2018). Data Mining para estudos de interação entre fármacos e previsão de Efeitos Adversos de Fármacos. Dissertação de Mestrado. Faculdade de Engenharia. Universidade do Porto. Porto, Portugal

Mitjà, O. \& Clotet, B. (2020) Use of antiviral drugs to reduce COVID-19 transmission. The Lancet Global Health, 8(5), e639-e640.

Pereira, A.S., Shitsuka, D.M., Parreira, F.J. \& Shitsuka, R. (2018). Metodologia da Pesquisa Científica. Universidade Federal de Santa Maria.

Pereira, MS (2020). Pandemia: os riscos da automedicação e os cuidados com a saúde. https://www.unisc.br/pt/noticias/pandemia-os-riscos-daautomedicacao-e-os-cuidados-com-a-saude

Wu, R., Wang, L., Kuo, H. C. D., Shannar, A., Peter, R., Chou, P. J., \& Kong, A. N. (2020). An update on current therapeutic drugs treating COVID-19. Current Pharmacology Reports 11, 1-15.

Retallack, H., Di Lullo, E., Arias, C., Knopp, K. A., Laurie, M. T., Sandoval-Espinosa, C., \& DeRisi, J. L. (2016). Zika virus cell tropism in the developing human brain and inhibition by azithromycin. Proceedings of the National Academy of Sciences, 113(50), 14408-14413.

Schmith, V. D., Zhou, J., \& Lohmer, L. R. (2020). The Approved Dose of Ivermectin Alone is not the Ideal Dose for the Treatment of COVID-19. Clinical Pharmacology \& Therapeutics. 108(4), 762-65.

Sousa, M. R. N., Barros, S. S., Silva, M., Oliveira, A. P. M., Rocha, G. M., \& Oliveira, G. A. L. (2020). Pathogenesis and treatment prospects for Covid-19: a review. Research, Society and Development, 9(7), 05973730.

Van, G. G., Wolterink, R. G. J., K., Elssen, C. H. M. J. V., Wieten, L., Germeraad, W. T. V., \& Bos, G. M. J. (2018). Influence of Vitamin C on lymphocytes: an overview. Antioxidants (Basel), 7(3).

World Health Organization. Folha informativa -COVID-19 (doença causada pelo novo coronavírus). https://www.paho.org/bra/index.php?option=com_conte nt\&view=article \&id=6101: covid19\&Itemid=875.

Wu, Y., Xu, X., Chen, Z., Duan, J., Hashimoto, K., Yang, L., \& Yang, C. (2020). Nervous system involvement after infection with COVID-19 and other coronaviruses. Brain, behavior, and immunity. 87, 18-22.

Zhai, P., Ding, Y., Wu, X., Long, J., Zhong, Y., \& Li, Y. (2020). The epidemiology, diagnosis and treatment of COVID-19. International journal of antimicrobial agents, 55(5). 105955.

Zhang, L., \& Liu, Y. (2020). Potential interventions for novel coronavirus in China: A systematic review. Journal of medical virology, 92(5), 479-490. 